\title{
Network Load Balancing in Dynamic Data Center
}

\author{
Shrikant Deshmukh ${ }^{1}$, Nitin Chopade ${ }^{2}$ \\ Department of Computer Science \& Engineering ${ }^{1,2}$, G. H. Raisoni College of Engineering and Management, \\ Amravati, India ${ }^{1,2}$ \\ Email:shrik240@gmail.com ${ }^{1}$, nitin.chopde@ raisoni.net ${ }^{2}$
}

\begin{abstract}
Distributed computing systems have become a natural setting in many environments for business and academia. This is due to the rapid increase in processor and/or memory hungry applications coupled with the advent of low-cost powerful workstations. Over the past three decade, parallel and distributed computing witnessed major growth due to the declined cost of hardware, advancement in communication technology, explosive growth of internet and need to solve large-scale problems. These systems often face the problem of load imbalance, which can degrade the performance of the system. Load balancing improves the system performance by dividing the work load effectively among the participating computers/nodes. In this paper proposed mobile virtual cloud network will be form and will make available to all nodes for execution but it is very critical to find the optimize node for the execution in the given network so that we are using bidding resource allocation algorithm. This will work like to the bidder. In this way we can form the virtual cloud network with total mobile cloud implementation.
\end{abstract}

Index Terms- Distributed computing, virtual cloud network, mobile cloud, mobile virtual cloud network

\section{INTRODUCTION}

Cloud computing is a computing paradigm. It has a large pool of systems that are connected in private or public networks. It provides dynamic infrastructure for applications, data and file storage. It is generally used in case of Internet. Users get service from a cloud without paying attention to the inner details. With the advent of this new technology, the cost of computation, application hosting, storage and delivery is reduced significantly. It provides the scalable resources such as applications and services, and the infrastructure on which they operate, on the Internet, as pay per-use basis to adjust the capacity quickly and easily.

Cloud computing is efficient and scalable but maintaining the stability of processing so many jobs in the cloud computing environment is a very complex problem with load balancing. Load balancing in cloud computing scenario has an important impact on the performance. A good load balancing makes this technology more efficient. It improves user satisfaction. Load balancing is a mechanism that distributes the excess dynamic local workload evenly over all the nodes. It makes sure that no single node is overwhelmed. Thus it improves the overall performance of the cloud computing system. Load balancing is a relatively new technique that facilitates networks and resources by providing a maximum throughput with minimum response time. A proper load balancing algorithm can help in utilizing the available resources optimally which thereby minimizes the resource consumption. Dividing the network traffic between servers can leads to data sent and received without major delay. Many different kinds of algorithms are available and used that helps to reduce traffic loaded between available servers. In this paper we proposed mobile virtual cloud network will be form and will make available to all nodes for execution but it is very critical to find the optimize node for the execution in the given network so that we are using bidding resource allocation algorithm. This will work like to the bidder. In this way we can form the virtual cloud network with total mobile cloud implementation.

\section{RELATED WORK}

Ali M. Alakeel [1] concentrates almost completely on the dynamic approach, due to its more realistic approach to load balancing. The aim of this paper is not to advance a specific dynamic load balancing policy, but rather to address the problem and present different approaches that have been used to develop a solution for it. Monika Kushwaha et. al. [2] suggested various aspects of load balancing and explains various load balancing approaches and strategies and their respective algorithms. The paper studied various policies which should be considered while designing the load balancing algorithm like information policy etc. Finally discussed Static and Dynamic load balancing strategies with their respective algorithms and concluded that each strategy has their own pros and cons and there exists no absolutely perfect balancing algorithm but one can use depending on the need. I.A. Botygin et. al. [3] proposed model experiment on approbation has shown the 


\section{Available online at www.ijrat.org}

effectiveness of the method of resources allocation. Presenting model experiment that used the method of dynamic connectivity of resources will be also effective in significantly increasing of the connected nodes and zoom in architecture. Deng Huafeng et. a. [4] proposed an algorithm for load balancing in the parallel and distributed systems. Firstly, all jobs are assigned to the machines according to classical Min-min algorithm. Then performance of the algorithm is evaluated by computing the fairness index. If the value of the fairness index is not within the proper range, an improvement algorithm can be executed over the intermedial results. During the process of the improvement algorithm, the machines are organized into pairs according to the total load of the tasks on them. Then each time no more than two tasks are allowed to be exchanged between each pair of machines to achieve better effect. The experimental results show that our algorithm performs efficiently and suffices the specific requirements of real-time systems. Suchitra Choudhary et. al. [5] proposed an method tondetects the malware and prevents the electronic devices from the malwares which can destroy or delete the data from electronic devices. In this paper, proposed an algorithm for the detection and prevention; we use set theory for detection and prevention the malwares.

\section{PROPOSED ARCHITECTURE AND METHODOLOGY}

In the proposed mobile virtual cloud network will be form and will make available to all nodes for execution but it is very critical to find the optimize node for the execution in the given network so that we are using bidding resource allocation algorithm. This will work like to the bidder. In this way we can form the virtual cloud network with total mobile cloud implementation.

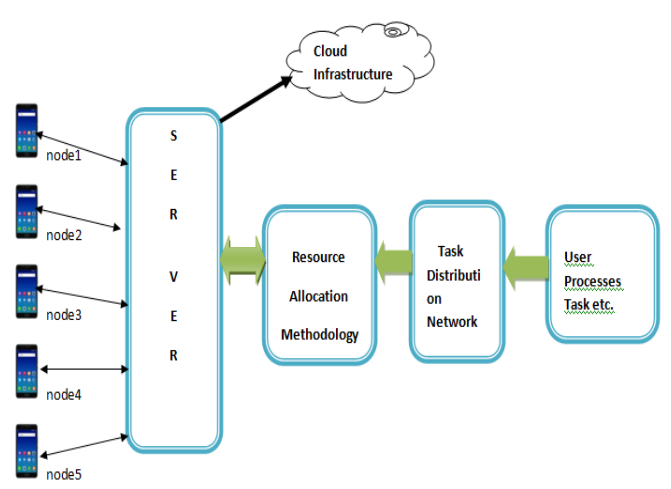

Figure 1: Proposed Architecture

The implementation is done on the resource availability of available resources in MCC. whenever the different node are available in network and to execute the certain task then finding out the efficient and optimized node is too much important in this we are implementing the architecture using agent based search mechanism which effectively help us in load balancing of in the allocation of the various task and manipulating the optimal resources

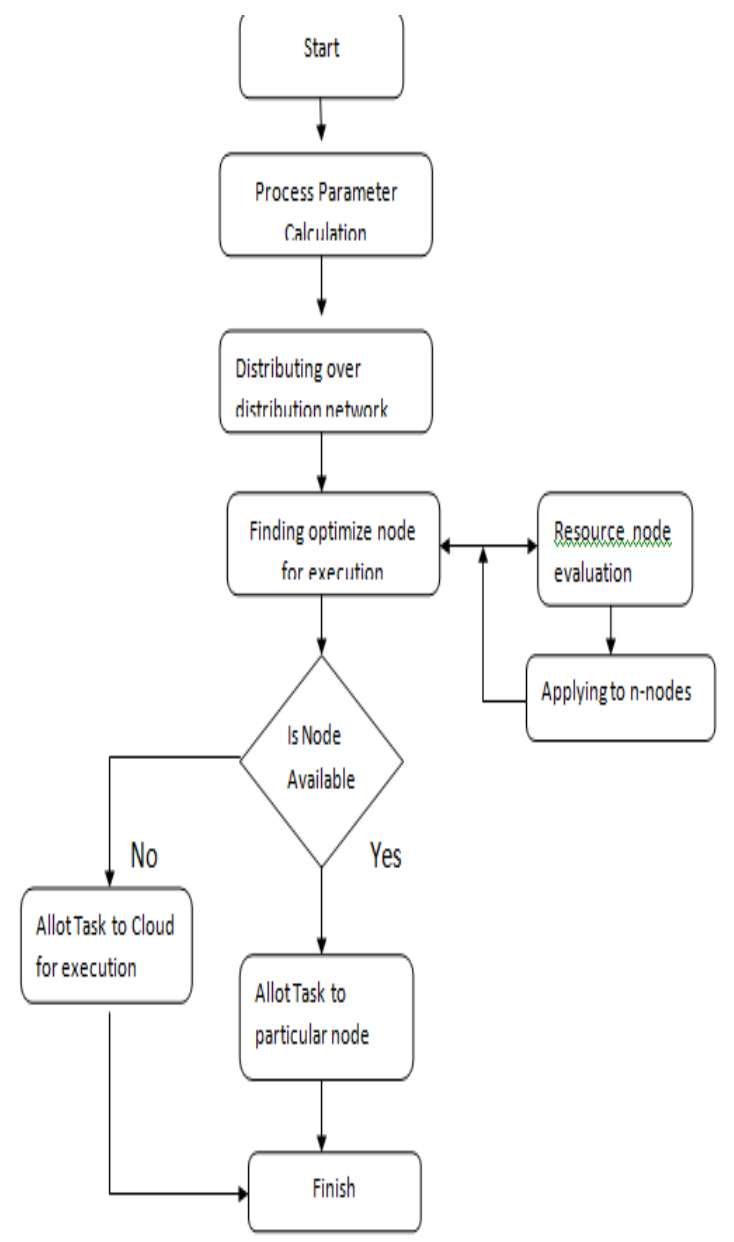

Figure 2: Proposed flowchart

In our proposed system the existing resources of cloud are taken in consideration for giving high performance to mobile and other devices which is cost consuming. So that it is possible to access the resources of joined node in the network in which the execution can be distributed to the another node and the device can be optimized. So that we are proposed the effective load balancing mechanism in support of the cloud in which the interconnected node can form the virtual cloud network. In the proposed mobile virtual cloud network will be form and will make available to all nodes for execution but it is very critical to find the optimize node for the execution in the given network so that we are using bidding resource allocation algorithm. This will work like to the bidder. In this way we can form the virtual cloud network with total mobile cloud implementation. 


\section{SIMULATION AND RESULT ANALYSIS}

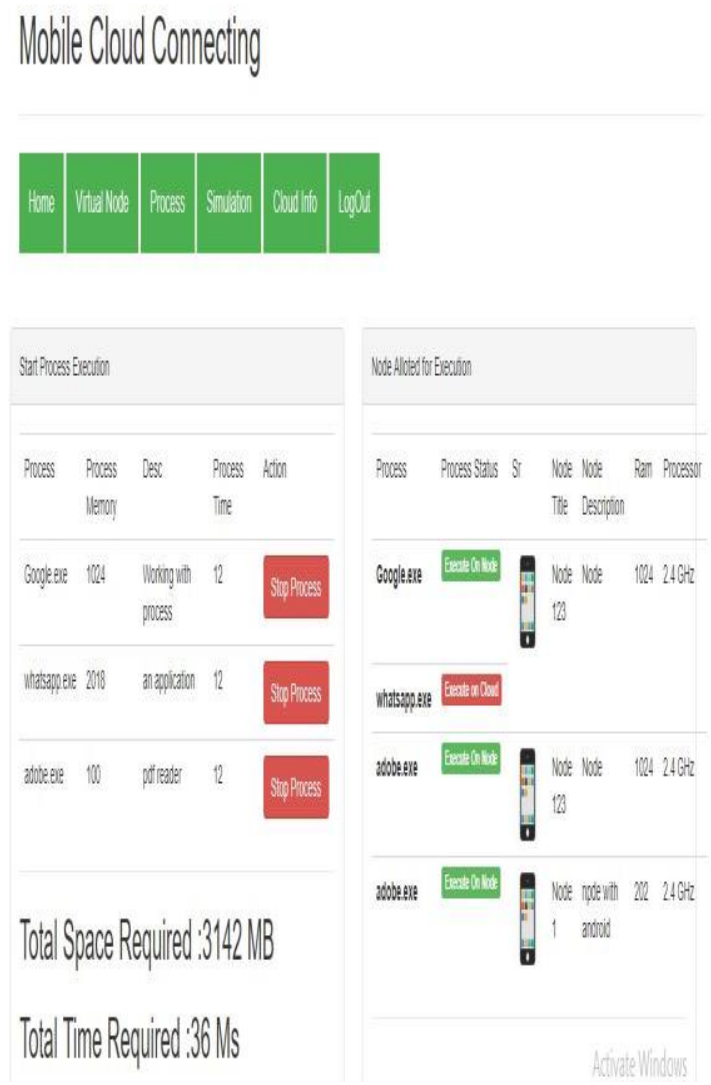

Figure 3: After the successfully login the first window is get visualize to the user in which the various process are get listed at the left side in which the console for the process will get started and according to that the working is going to implemented .In this the demonstration of complete working of algorithm is get implemented for the efficient.

\section{Mobile Cloud Connecting}

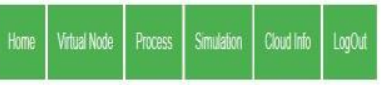

\section{Add Virtual Node Here}

\begin{tabular}{|c|c|}
\hline 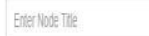 & \\
\hline 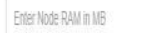 & \\
\hline 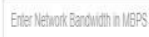 & \\
\hline Sset Dence 0s- & , \\
\hline Seted Denic Powessy- & , \\
\hline 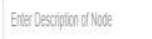 & \\
\hline Sitont & Atrivate Wirdous \\
\hline
\end{tabular}

Figure 4: The demonstration is going to be workout onto the virtual network in which the various nodes are getting created virtually so that the current window will help the user to create $n$ number of nodes for the execution. This will help to increase it to more extend.
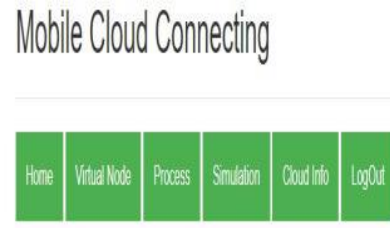

\begin{tabular}{|c|c|c|c|c|}
\hline No6e The & 10640 Dercitition & Ram & Prowsor & Stats \\
\hline Whe tiz & Node & 1024 & 24GHz & uphete \\
\hline Node1 & nper whit andoid & 202 & 246 Hz & Unter \\
\hline
\end{tabular}

Figure 5: This is terminal where node related information can be rectify. Here the update option is making available to the node to make information more effective. In this user can alter the information as per the requirement.
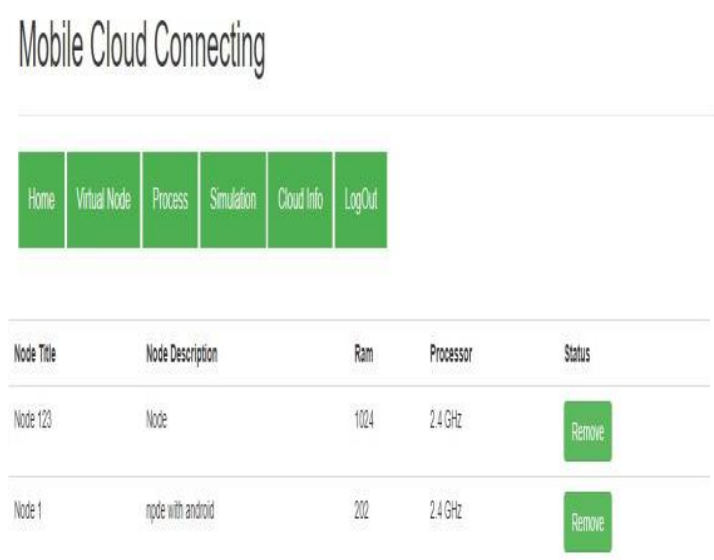

Figure 6: Here the access for removing node will get provided in which the created node can be deleted by clicking on to the remove option this will permanently delete node from simulator that get develop. Once it Will not accessible latterly. 


\section{Mobile Cloud Connecting}

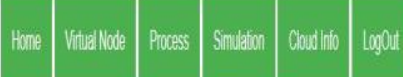

\begin{tabular}{|c|c|c|c|c|}
\hline Node Ttes & Node Description & Ram & Processor & Status \\
\hline Node 123 & Node & 1024 & 24GHz & Viakelandive \\
\hline Node 1 & rode wha andocid & 202 & $24 \mathrm{GHz}$ & Intre landive \\
\hline
\end{tabular}

Figure 7: This window will help the user to activate and deactivate the node execution in this the option is provided to make the node active or inactive. The activate node are taken for consideration only. This will help the user to manipulate node functionality quickly.

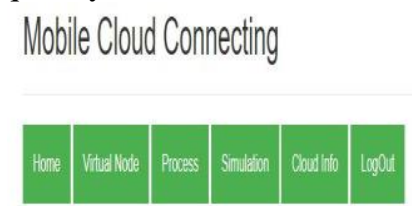

\section{Add Processor Here}

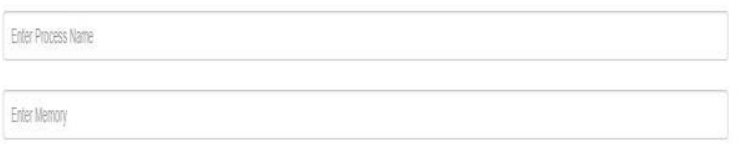

Eter Dessionon Pincess

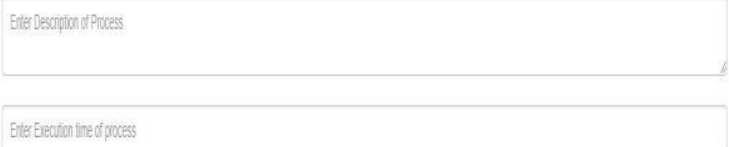

Ethe Eeroun inedifous

Adi Proms

Figure 8: Here user can create the process as per the requirement this will helpful for creating the process virtually here are the different options are get provided like process name, space required by the process, description of the process and execution time as well.

\section{Mobile Cloud Connecting}

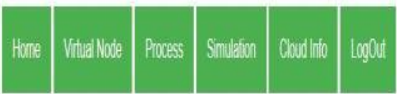

\begin{tabular}{|c|c|c|c|c|}
\hline Process Tite & Process llamory & Process Description & Tme Required & Action \\
\hline Gompere & 1024 & Working uith process & 12 & upetitrouess \\
\hline intatsappere & 2018 & an zeccication & 12 & Uphate Prows \\
\hline abobere & 100 & peff teaber & 12 & Upatel Process \\
\hline
\end{tabular}

Figure 9: This window get provided for updating the process if any mistake has been done while uploading. Here is terminal get provided for updating the process in proper way. It will rectify the committed mistakes.

\section{Mobile Cloud Connecting}
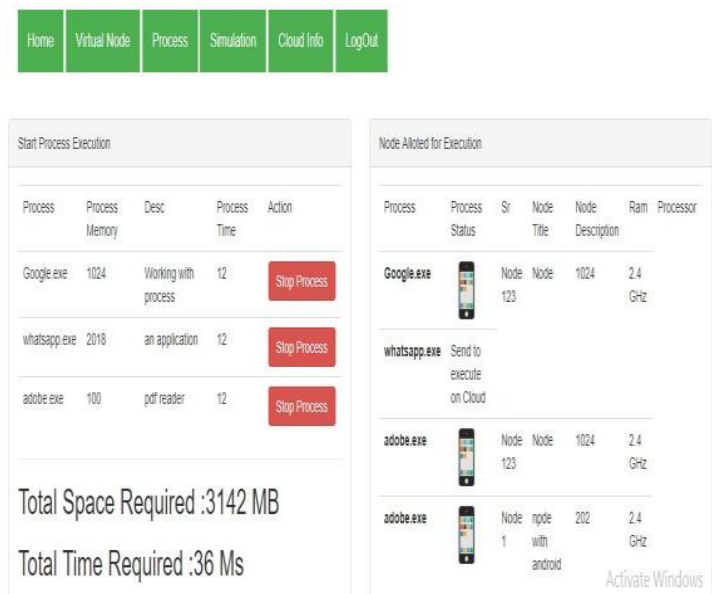

Figure 10: This the actual working of the project working explain over here in which the algorithmic work is getting workout step by step it will show the dynamic allocation of the process according to node requirement. Which will explain complete working of proposed work.

\section{RESULT EVALUATION}

We collect the result of our proposed system on some parameters. We implement the proposed to balance the data of network, in existing the resources of cloud are taken in consideration for giving high performance to mobile and other devices which is cost consuming. So that it is possible to access the resources of joined node in the network in which the execution can be distributed to the another node and the device can be optimized. So that we are proposed the effective load 


\section{Available online at www.ijrat.org}

balancing mechanism in support of the cloud in which the interconnected node can form the virtual cloud network. In the proposed mobile virtual cloud network will be form and will make available to all nodes for execution but it is very critical to find the optimize node for the execution in the given network so that we are using bidding resource allocation algorithm. This will work like to the bidder. Load balancing plays an important role in managing available resources. The designs that rely on remote cloud services, however, sometimes overlook the abundant resources (e.g., storage, communication, and computation) on mobile devices. In particular, when the remote cloud services are unavailable (due to service downtime or network issues), these smart devices can no longer working.

One can think of proposed system as a set of representations of an existing (or future) system. These representations initially describe a general, high-level functional organization, and are progressively refined to more detailed and concrete descriptions. Proposed system conveys the informational content of the elements consisting of a system, the relationships among those elements, and the rules governing those relationships. In proposed system we create a node in which the process should be run on the basis of the memory. If the memory of process is near by the node then that process run in that node otherwise the process run in the cloud. For the executing the process on node we need the node in active mode, if the node is not active then we need to make active that node. Evaluation of the proposed system focuses on to balance the load of the network.

The process which are created by the user for the execution. The process contain the task which store some information or data of the user, when the process is created the main work is to run that process and for that we have start button to run that process and if we want to stop that process we have stop button to stop that running process. We have four processes with their process memory and in table we can see that all the available processes are getting started for execution.

Running Position of Process

\begin{tabular}{|c|c|c|}
\hline Process & $\begin{array}{c}\text { Process Memory in } \\
\text { MB }\end{array}$ & Status \\
\hline google.exe & 700 & Started \\
\hline adobe.exe & 900 & Started \\
\hline amazon.exe & 1100 & Started \\
\hline yahoo.exe & 1200 & Started \\
\hline
\end{tabular}

The node contain the memory which store some information or data of the user, for balancing the data we need to make the node active which are inactive mode. To activate that node which we want to allot should be activate by clicking on active button.

Available Nodes

\begin{tabular}{|c|c|c|}
\hline Node Title & $\begin{array}{c}\text { Node Memory in } \\
\text { MB }\end{array}$ & Status \\
\hline Node1 & 1100 & Active \\
\hline Node2 & 950 & Active \\
\hline Node3 & 800 & Active \\
\hline Node4 & 400 & Active \\
\hline
\end{tabular}

The actual result of our proposed system is shown in which have three processes and only two nodes are in active state, when we run our process at that time the memory of process can allocate the data to the nearby node by using the memory allocation of the node. The nearby node can be allocated by the nearby process memory. The important thing is allocation time which is less than other system for that we can used time allocation technique to execute the processes.

Allocation Time by Proposed System

\begin{tabular}{|c|c|c|c|c|}
\hline Process & $\begin{array}{c}\text { Process } \\
\text { Status } \\
\text { d Node }\end{array}$ & $\begin{array}{c}\text { Allotte } \\
\text { ma }\end{array}$ & $\begin{array}{c}\text { Allocat } \\
\text { ion } \\
\text { Time( } \\
\text { ms) }\end{array}$ \\
\hline $\begin{array}{c}\text { google.e } \\
\text { xe }\end{array}$ & $\begin{array}{c}\text { Executed } \\
\text { on Node }\end{array}$ & Node3 & 700 & 20 \\
\hline $\begin{array}{c}\text { adobe.e } \\
\text { xe }\end{array}$ & $\begin{array}{c}\text { Executed } \\
\text { on Node }\end{array}$ & Node2 & 900 & 30 \\
\hline $\begin{array}{c}\text { amazon. } \\
\text { exe }\end{array}$ & $\begin{array}{c}\text { Executed } \\
\text { on Node }\end{array}$ & Node1 & $\begin{array}{c}110 \\
0\end{array}$ & 40 \\
\hline $\begin{array}{c}\text { yahoo.e } \\
\text { xe }\end{array}$ & $\begin{array}{c}\text { Executed } \\
\text { on Cloud }\end{array}$ & Cloud & 409 & 90 \\
\hline \multicolumn{5}{|c|}{ Total Estimated Time } \\
\hline
\end{tabular}

\section{CONCLUSION}

Efficiently exploiting the mobile devices' idle computing, storage, and sensing capacity can greatly improve the quality of service provided by virtual mobile cloud computing. To achieve this goal, an appropriate architecture of virtual mobile cloud computing and a dedicated scheduling algorithm are considered important. To address these issues, this paper contributes in several ways by providing suitable definitions of critical aspects and proposing efficient algorithms and approaches. This paper has attempted to present the most recent ideas and achievements realized in load balancing in mobile virtual cloud systems. In our research proposed an 


\section{Available online at www.ijrat.org}

mobile virtual cloud network will be form and will make available to all nodes for execution but it is very critical to find the optimize node for the execution in the given network so that we are using bidding resource allocation algorithm. This will work like to the bidder.

\section{REFERENCES}

[1] Ali M. Alakeel, "Load Balancing in Distributed Computer Systems", (IJCSIS) International Journal of Computer Science and Information Security, Vol. 8, No. 4, 2010.

[2] Monika Kushwaha, Saurabh Gupta, "Various Schemes of Load Balancing in Distributed Systems- A Review", International Journal of Scientific Research Engineering \& Technology (IJSRET), ISSN 2278 - 0882, Volume 4, Issue 7, July 2015.

[3] I.A. Botygin, A.E. Nagiyev, "Model Experiment for Load Balancing in a Distributed Computer System" IEEE, International Conference on Mechanical Engineering, Automation and Control Systems (MEACS), 2015.

[4] Deng Huafeng, Zhong Linhui, Ye Maosheng, "An Efficient Load Balancing Algorithm in Distributed Systems", IEEE, International Forum on Information Technology and Applications, 2010.

[5] Suchitra Choudhary1, Khaleel Ahmad2, Jayant Shekhar3,"Load Balancing in Distributed System through Task Migration", International Journal of Engineering and Technology (IJET), Vol 5 No 2 Apr-May 2013.

[6] Aly E. El-Abd, "Load Balancing in Distributed Computing Systems Using Fuzzy Expert Systems", TCSET'2002, February 18-23,2002.

[7] XianglinWei,1 Jianhua Fan,1 Ziyi Lu,1 and Ke Ding2, "Application Scheduling in Mobile Cloud Computing with Load Balancing", Hindawi Publishing Corporation, Journal of Applied Mathematics, Volume 2013, Article ID 409539, 13 pages http://dx.doi.org/10.1155/2013/409539.

[8] Rajwinder Kaur1 and Pawan Luthra2, "Load Balancing in Cloud Computing", Proc. of Int. Conf. on Recent Trends in Information, Telecommunication and Computing, ITC, Association of Computer Electronics and Electrical Engineers, 2014.. 